\title{
Adaption agiler Prinzipien für den Hochschulkontext am Beispiel des Universitätskollegs der Universität Hamburg
}

\author{
Kerstin Mayrberger $^{1}$ (D) $\cdot$ Markus Slobodeaniuk $^{1}$
}

Online publiziert: 11. August 2017

(C) Der/die Autor(en) 2017. Dieser Artikel ist eine Open-Access-Publikation.

Zusammenfassung Dieser Praxisbericht diskutiert die Relevanz agiler Prinzipien anhand des Fallbeispiels „Universitätskolleg“, einer zentralen Organisationseinheit zur Verbesserung von Studium und Lehre an der Universität Hamburg. Mit besonderem Fokus auf dem Rahmenwerk Scrum und der Methode Lean Kanban werden bisherige Entwicklungen beschrieben, Chancen und Herausforderungen agiler Prinzipien im Hochschulkontext exemplarisch benannt und reflektiert sowie erste Schlussfolgerungen für nächste Prozessschritte gezogen.

Schlüsselwörter Agilität · Lehrentwicklung · Organisationsentwicklung · Scrum · Kanban · Universität · Change Management

\section{Adaption of agile principles in university context using the example of Universitätskolleg at Universität Hamburg}

\begin{abstract}
This report on practice discusses the pertinence of agile principles on the basis of the case "Universitätskolleg", a central organisational unit of Universität Hamburg to promote the quality of studies and teaching. It describes previous experience with the framework scrum and the method Lean Kanban in mind. Odds and challenges of agile prin-
\end{abstract}

Prof. Dr. Kerstin Mayrberger

kerstin.mayrberger@uni-hamburg.de

Markus Slobodeaniuk

markus.slobodeaniuk@uni-hamburg.de

1 Universitätskolleg, Universität Hamburg, Schlüterstr. 51, 20146 Hamburg, Deutschland ciples in university context are described and reflected and next steps are named.

Keywords Agility - Teaching development . Organisational development · Scrum · Kanban · University · Change mangement

\section{Das Universitätskolleg als Modellversuch}

Der vorliegende Beitrag referenziert auf ein Praxisbeispiel aus dem universitären Kontext, das die Verbesserung von Studium und Lehre als Auftrag hat. Somit ist die Gestaltung von Wandel die tägliche Aufgabe. In diesem Rahmen sind daher die Interaktionen mit den unterschiedlichen Akteuren von Studierenden über Lehrenden und Personen aus der Administration (u. a. Lehre und Studium, Öffentlichkeitsarbeit sowie IT-Services) hinweg über Mikro- und Mesoebene besonders bedeutsam. Das Universitätskolleg (vgl. https://www.universitaetskolleg.de) besteht seit Anfang 2012, wird aus Mitteln des „Qualitätspakt Lehre“ des Bundesministeriums für Bildung und Forschung (BMBF) gefördert und ist mittlerweile eine zentrale Organisationseinheit der Universität Hamburg. ,Als Bildungseinrichtung, Entwicklungs- und Experimentierlabor, Kommunikations-, Koordinations- und Kooperationsplattform und als Thinktank zur wissenschaftlichen Absicherung und kritischen Begleitung sollte das Universitätskolleg für alle den Übergang aus Schule und Beruf in die Universität betreffenden Aktivitäten, Maßnahmen und Initiativen wirken (...), um auf diese Weise zu einer grundlegenden und nachhaltigen Verbesserung von Lehre und Studium in einer Phase beizutragen, in der entscheidende Weichenstellungen für den Studienerfolg vorgenommen werden“ (vgl. Universitätskolleg 2017, S. 16). Von 2017 bis 2020 wird dieses Projekt als ,Modell- 
versuch Universitätskolleg 2.0“ mit fast 11 Mio. Euro in der zweiten Runde weitergefördert. Die zuvor über 40 zumeist dezentral angesiedelten und agierenden Teilprojekte wurden in einem partizipativen Prozess mit den Fakultäten auf eine überschaubare Zahl von Maßnahmen reduziert, die sich nunmehr auf alle Übergangssituationen im Sinne kritischer Bildungspassagen im Studium beziehen (u. a. Selbsteinschätzung, Willkommenskultur, Studentische Beteiligung, Schreibzentrum, Lehrlabor und extracurriculares Kursangebot). Eine wichtige Erkenntnis aus dem Universitätskolleg 1.0 war vor allem, dass die hohe Disparität sowie die mangelnde Konsistenz der Angebote zu verhältnismäBig geringer Akzeptanz bei Studierenden und Lehrenden führt. Ebenso machten vielfältige individuelle Lösungsansätze eine hochschulweite Verstetigung nahezu unmöglich. Insofern ist für das Universitätskolleg 2.0 die Idee leitend, die erfolgreichen Ansätze aus der ersten Phase weiter auszuarbeiten und in der zweiten Phase nunmehr auf möglichst viele der acht Fakultäten wirksam auszuweiten und dabei alle relevanten Stakeholder möglichst stetig und zeitnah einzubeziehen, um deren (fach-)spezifischen Bedarfe in die Entwicklung der unterschiedlichen Angebote ebenso stetig und zeitnah einfließen zu lassen und damit idealerweise die spätere Akzeptanz der Maßnahmen zu erhöhen. Eine solche Zielsetzung stellt in einem klassischen Projektmanagement mit einem vorab antizipierten Meilensteinplan sowie einer klassischen Teamstruktur eine Herausforderung dar und widerspricht gar der Zielsetzung. Daher wird als Teil des Modellversuchs Universitätskolleg 2.0 unter nunmehr erweiterter Zielsetzung auch die Erprobung veränderter in diesem Fall agiler - Organisationsprinzipien vorgenommen, die potenziell den Dynamiken der Bedarfe und damit einer nachhaltigeren Verankerung von Lehrentwicklungsmaßnahmen stärker dienen, als es die bisherigen Erfahrungen zum Projektmanagement aus der Hochschule zeigen (Hanft et al. 2016, 2017).

\section{Agile Prinzipien und Scrum}

Die Arbeit im Team nach agilen Prinzipien ist ein schon lange bekanntes Vorgehen aus der Softwareentwicklung. Der Ursprung von ,agil“" wird in der Formulierung eines ,Agile Manifesto“ (vgl. http://agilemanifesto.org/) 2001 gesehen, das für folgende Kernwerte plädiert:

- „Individuen und Interaktion stehen über Prozessen und Werkzeugen“"

- Funktionierende Software steht über umfangreicher Dokumentation

- die Zusammenarbeit mit dem Kunden steht über der Verhandlung von Verträgen
- das Reagieren auf Veränderungen steht über dem Befolgen eines Plans.

Das bedeutet: „Obwohl die Dinge auf der rechten Seite ihren Wert haben, messen wir den Dingen auf der linken Seite größeren Wert bei“ (Übersetzung nach Gloger 2016, S. 20).

Mittlerweile werden entsprechende Prinzipien und Ansätze in andere Organisationsbereiche und auf Themen übertragen, in denen Menschen gemeinsam prozessorientiert an Produkten im weitesten Sinne arbeiten und handelnd tätig werden. So sind für den Bildungsbereich bereits Ausführungen zu agilen Prinzipien für die Zusammenarbeit von Schüler/innen (vgl. http://agile-education.de/ oder http://eduscrum.nl), didaktische Überlegungen zur Rolle von agilen Prinzipien für das Lernen und Lehren mit digitalen Medien an der Hochschule (Mayrberger 2017) sowie Folgerungen für eine erfolgreiche handlungsorientierte Implementierung von Lehrentwicklungsvorhaben (Hanft et al. 2017) zu finden.

Das Rahmenwerk „Scrum“ wurde im Nachgang zum Agilen Manifest von Jeff Sutherland, Ken Schwaber und Mike Beedle für den Kontext der Softwareentwicklung publiziert (Schwaber et al. 2001; vgl. für Scrum als Bewegung ausführlicher Gloger 2016). Nachfolgend wird dieses Rahmenwerk in seinen Grundzügen knapp skizziert und flankierend Bezug auf konzeptionelle Überlegungen zur Realisierung dieser Prinzipien im Universitätskolleg der Universität Hamburg genommen. Dabei können die eingangs aufgezeigten relevanten Akteure an der Hochschule hier äquivalent zu den im Rahmenwerk benannten Stakeholdern oder Kunden betrachtet werden.

In Anlehnung an den deutschsprachigen Scrum-Guide (Schwaber und Sutherland 2016) ist Scrum(n) ein „Rahmenwerk, innerhalb dessen Menschen komplexe adaptive Aufgabenstellungen angehen können und durch das sie in die Lage versetzt werden, produktiv und kreativ Produkte mit höchstmöglichem Wert auszuliefern“ (ebd., S. 3). Häufig wird zusätzlich auf „Lean-Kanban“ zur Unterstützung von Scrum zurückgegriffen und mit sogenannten „Kanban-Boards“ gearbeitet (deren einfachste Spaltenunterteilung zwischen „,todo“, „doing“ und ,done“ unterscheidet), weshalb für diesen Fall von „Scrumban“ gesprochen wird. Die Methode Lean-Kanban ist ein hochflexibles und adaptierbares Steuerungsinstrument für die Wissensarbeit und deren zielgerichtete Prozesssteuerung und entwickelt sich im Idealfall evolutionär und in iterativen Schleifen mit dem Team. In diesem Prozess wird eine Balance zwischen gesundem Arbeitstempo, einem effizienten Arbeiten und keiner Verschwendung von Ressourcen angestrebt. Kernelement jeder Variante des agilen Projektmanagements ist dabei immer die Begrenzung des Planungs- und Steuerungsaufwands zum Projektvorgehen auf kurze Iterationen, in 
Scrum als „Sprints“ mit einer Dauer von maximal vier Wochen bezeichnet. Das Ergebnis jedes Sprints muss eine erweiterte Version des für den Kunden nutzbaren Projektzwischenergebnisses, des „Inkrements“, sein. Durch die kurzen Zeiträume in der Realisierung und das gemeinsame Prüfen von Zwischenergebnissen mit dem Kunden bzw. den Stakeholdern erlaubt das agile Vorgehen eine frühzeitige Anpassung an veränderte Umgebungsanforderungen, die ansonsten das gesamte Projektergebnis gefährden könnten.

Bevor das Rahmenwerk Scrum nunmehr auf passende Maßnahmen im Universitätskolleg 2.0 angewendet wird, wurde das Rahmenwerk Scrum in Kombination mit Kanban als Scrumban bereits im Kontext der Implementierung von IT-Anwendungen noch im Universitätskolleg 1.0 erprobt. Hierbei wurde im Sinne des Scrum-Rahmenwerks mit dem entsprechenden Rollenkonzept gearbeitet. Das Rollenkonzept bei Scrum umfasst exakt nur drei Rollen, aus denen ein Scrum-Team besteht: das selbstorganisierte, crossfunktionale Entwicklungsteam bestehend aus drei bis neun Mitgliedern, einem Scrum-Master und einem Product Owner (vgl. Gloger 2016; Schwaber und Sutherland 2016).

- Die Rolle des „Product Owner“ ist für die Wertmaximierung des Produkts im Sinne der Stakeholder verantwortlich und formuliert die Produktvision. Hierbei handelt es sich um eine Person (und kein Komitee oder Gremium), die in direktem Kontakt mit den Stakeholdern produktbezogene Bedarfe abholt, deren Priorisierung im Sinne des definierten Produkterfolgs sowie der Produktvision vornimmt und diese in einem „Backlog“, einer öffentlich zugänglichen Übersicht aller bekannten Anforderungen, dokumentiert. Gegenüber dem Entwicklungsteam nimmt der Product Owner keine Leitungsfunktion wahr - er oder sie steuert das Projektvorgehen nur durch die Vorgabe, ,was“ als nächste Anforderung(en) aus dem Backlog aufgrund hoher Priorisierung umgesetzt werden sollte.

- Das „Entwicklungsteam“ entscheidet über das „Wie“ der Umsetzung von Anforderungen zur Bereitstellung eines Produkts und den Weg zum jeweiligen Sprintergebnis in Form eines potenziell auslieferbaren fertigen Inkrements. Es besteht idealerweise aus Personen mit fachlichem Tiefenwissen sowie mit vielseitigen Kompetenzen in der Breite (sogenannte ,T-Shaped Professionals“) und bildet ein crossfunktionales, selbstorganisiertes Team.

- Der „Scrum-Master“ ist dafür verantwortlich, dass das Team ein Verständnis für Scrum aufbaut und Prozesse im Sinne des Rahmenwerks durchgeführt werden. Dazu gehört die Vorgabe von „time-boxed events“ wie dem täglichen kurzen „Daily Scrum“, dem spätestens alle vier Wochen stattfindenden „Sprint Planning“, dem „Sprint Review“ (mit den Stakeholdern) sowie der ,Sprint Retrospektive“. Er oder sie sorgt dafür, dass das Scrum-Team die Praktiken und Regeln von Scrum einhält, und hält dem Entwicklungsteam den Rücken frei, indem er/sie versucht, „Impediments“, d. h., soziale, kommunikative, administrative oder organisatorische Hindernisse aller Art, für das Team und mit dem Team zu beseitigen. Die Rolle des Scrum-Masters ist ein „Servant Leader“ für das Scrum-Team insgesamt. Entsprechend wirbt er auch für Verständnis für die Arbeitsweise nach Scrum nach außen hin, damit das Team in den geregelten Abläufen nicht gestört wird, und hilft so dabei, dass das Team sich auf sich selbst und die Entwicklung einer optimalen $\mathrm{Zu}$ sammenarbeit konzentrieren kann, was sich schließlich in einem sehr guten Produkt im Sinne der Produktvision für das jeweilige Projekt niederschlagen sollte.

Das Scrum-Team arbeitet in seinen drei Rollen im Idealfall selbstorganisiert und interdisziplinär und kann so die eigene Flexibilität, Kreativität und Produktivität optimieren. Dazu baut Scrum Referenzwerte auf, die die Grundidee dieses Rahmenwerks fassbar machen: „Wenn die Werte Selbstverpflichtung, Mut, Fokus, Offenheit und Respekt durch das Scrum-Team verkörpert und gelebt werden, werden die Scrum-Säulen Transparenz, Überprüfung und Anpassung lebendig und bauen bei allen Beteiligten Vertrauen zueinander auf" (Schwaber und Sutherland 2016, S. 4).

\section{Anwendung von agilen Prinzipien und Scrum im Universitätskolleg}

Den Prinzipien agilen Projektmanagements und gar dem Rahmenwerk Scrum bzw. Scrumban wurde und wird grundsätzlich auch im Universitätskolleg in Maßnahmen gefolgt, in denen diese zum Gegenstand und zum Team passen. Damit sich alle Teammitglieder und interessierten Stakeholder mit entsprechendem Wissen und Können und idealerweise auch Wollen einbringen können, wurden zu Beginn der Projektphase des Universitätskolleg 2.0 systematisch passgenaue Schulungen von unterschiedlicher Dauer über einen externen Anbieter zu Scrum und Kanban angeboten, die alle (in Abstimmung mit der Personalentwicklung) Teil der Arbeitszeit waren.

Nachfolgend werden entsprechende Erfahrungen in der Anwendung im Universitätskolleg über die bisherige $\mathrm{Ge}-$ samtlaufzeit seit 2012 dargestellt.

Im Universitätskolleg 1.0 im Teilprojekt für IT-Konzepte gestaltete sich das Vorgehen nach agilen Prinzipien exemplarisch wie folgt: Es wurden sowohl Treffen mit Stakeholdern in den Fakultäten und Einrichtungen wie auch die internen Abläufe des Teams in zeitfixierten Sprints von zwei Wochen organisiert. In Anlehnung an Scrum arbeitete das Team dem Projektziel durch iterative Projektabschnitte (Sprints) mit festen Ritualen (sprint planning, sprint re- 
view und sprint retrospective) entgegen. Zur Umsetzung einzelner Aufgaben integrierte es Beschränkungen basierend auf dem Kanban-Konzept durch die Einführung eines „Work in Progress"-Limits von maximal der Anzahl der Teammitglieder. Das „Work in Progress"-Limit (WiP-Limit) beschränkt die Anzahl parallel begonnener Aufgaben und sorgt somit dafür, dass nicht zahlreiche Aufgaben durch einzelne Teammitglieder begonnen werden, die dann jedoch innerhalb des festgesetzten zeitlichen Terminrahmens des Sprints nicht umgesetzt werden, wodurch am Ende des Sprints eine Reihe offener Aufgaben besteht, jedoch kein neues Ergebnis. Die Herbeiführung eines (potenziell releasefähigen) neuen Produktstatus als eines der wichtigsten Prinzipien von Scrum betrachtete das Team dabei immer aus der Kundenperspektive, verbunden mit der Frage, welche Fortschritte eigener Arbeit sofort zur Verbesserung der Informationssicht auf das (neue) Produkt wahrgenommen werden können.

Dieses Team aus dem Universitätskolleg 1.0 zeichnete sich dadurch aus, dass neben den Grundkenntnissen zu Scrum und Kanban, die vor allem den studentischen Mitarbeitenden im Team als Theoriewissen aus dem Studium bekannt waren und im Projekt erstmalige praktische Anwendung fanden, sich das Team gemeinsam in dieses Vorgehensmodell einarbeitete und mit deren Anpassung an den Hochschulbereich auf Grundlage von Reddys (2015) Buch „The Scrumban [R]Evolution: Getting the Most Out of Agile, Scrum, and Lean Kanban“ experimentierte. Für das Team war es in der Anfangsphase eine starke Umgewöhnung, Aufgaben eigenverantwortlich zu „ziehen“ und nicht zugewiesen zu bekommen, die Kommunikation im Team stärker auszubauen und Informationen über den Stand der eigenen Aufgaben stärker als gemeinsame Wissensgrundlage für alle Teammitglieder ständig verfügbar zu halten. Während der Start eines neuen Sprints mit der Planungsphase (sprint planning) sehr schnell zu produktiver Projektorganisation mit fest definiertem Sprintziel und einer Aufgabenliste (sprint backlog) führte, bereitete die Präsentation des Sprint-Ergebnisses im abschließenden ,sprint review“ oft gemeinsam mit Stakeholdern - deutlich mehr Schwierigkeiten. Es wurde die Erfahrung gemacht, dass anstelle der Vorstellung des neuen Produktergebnisses sich das Team hier oft auf die Vorstellung der eigenen Tätigkeiten konzentrierte. Die Bedeutung und Rolle der anwesenden Stakeholder im Produktentwicklungsprozess einzuschätzen und schätzen zu lernen gehört zu den Erfahrungen, die im klassischen Projektmanagement weniger zum Tragen kommen. Eine weitere wichtige Erfahrung aus diesem Beispiel für die Organisation von Projekten auch im Hochschulumfeld ist die Durchführung der ,sprint retrospectives“ im Anschluss an das „sprint review“. Dieses wird üblicherweise nur im internen Teamkreis durchgeführt und ist der Ort, an dem die oben genannten Referenzwerte für die Teament- wicklung besonders zum Tragen kommen (können). Hier diskutiert das Team über den Verlauf der Aufgabenumsetzung im vergangenen Sprint und geht vor allem auf die $\mathrm{Zu}$ sammenarbeit im Team ein. Auf diese Weise kann das Team auch emotionale Störfaktoren ansprechen, die die Teamzusammenarbeit behindern können oder konkret behindert haben, jedoch im „Normalbetrieb“ nicht offen ausgesprochen werden.

Auf Basis dieser ersten Erfahrungen im Universitätskolleg 1.0 wurden für den nachfolgenden ,Modellversuch Universitätskolleg 2.0“, der sich hin zu knapp zehn zentral organisierten und kondensierten Maßnahmen weiterentwickelt hat, gefolgert, innerhalb des Modellversuchs (2017-2020) organisatorische Aufgabenteilungen zwischen den Teams im Sinne der agilen Prinzipien zu erproben. Nachfolgende Beispiele illustrieren Erfahrungen der ersten Monate schlaglichtartig:

- Durch ein zentrales Redaktionsteam werden die Mitarbeitenden der Einzelmaßnahmen von der Bearbeitung der Website, von der Erstellung von Flyern und Plakaten (inkl. der Logistik zur Verteilung) sowie sonstiger Publikationen freigestellt. Dies bezieht sich natürlich nur auf den grafischen Entwurf, die Korrektur und die Produktion, nicht auf die Erstellung der (Fach-)Inhalte.

- Die an der zentralen Organisationseinheit bereits verankerte IT-Administration unterstützt die Maßnahmen in der IT-Basisversorgung (Geräteausstattung, Software). Sie wird durch das Team DevOp (kurz für Development and Operations) erweitert, das gemeinsam mit den Maßnahmen an digitalen Lösungen arbeitet wie beispielsweise mit dem Team Schreibzentrum oder dem Team Selbsteinschätzung notwendige Softwareprodukte definieren, anpassen (Development) und betreiben (Operations).

- Ebenfalls bereits in der zentralen Organisationseinheit verankert ist das Team Ressourcen-Management (ReM), welches die Maßnahmen wie das Schreibzentrum, das Lehrlabor oder die Kursangebote bei der Einstellung von Studentischen Hilfskräften und TutorInnen, der Vergabe von (Lehr-)Aufträgen und sonstigen Personalaufgaben unterstützt.

- Ein Team Cluster-Koordination übernimmt neben übergreifenden Moderationsaufgaben für die Teams in den Maßnahmen beispielsweise auch die Verwaltung von extracurricularen Angeboten, die im universitätsweiten Campus Management System hinterlegt werden.

- Nach und nach bilden sich spezifische Varianten von Stakeholder-Gruppen heraus wie beispielsweise für die Maßnahme Selbsteinschätzung unter starkem Einbezug von Studienbüroleitungen oder in Form eines professoralen Expertenrats beim fakultätsübergreifenden Schreibzentrum, die in je spezifischer Weise den Teams Rück- 
meldungen zu den bisherigen Produkten und zu weiteren Bedarfen geben.

Die hier benannten Teams in den Maßnahmen sind mit einem Entwicklungsteam gleichzusetzen, die sich aufgrund der übergreifenden Unterstützung im Sinne von der Delegation von Impediments auf ihre jeweilige Fachaufgabe und deren operative Umsetzung von Angeboten sowie den Kontakt zu Lehrenden und vor allem Studierenden als Hauptzielgruppe konzentrieren können. Die Unterstützung von Teams durch eine Prozessbegleitung (,Scrum-Master“) wird als Element der Qualitäts- und Ergebnissicherung ebenfalls wertgeschätzt, allerdings häufig noch fälschlicherweise mit einer Leitungsaufgabe verbunden. Die Aufteilung der klassischen Projektleitungsaufgaben auf zwei getrennte Personen (Scrum-Master und Product Owner) unter gleichzeitiger Stärkung der Eigenverantwortung des Teams für den Projekterfolg beim agilen Projektmanagement sollte an Hochschulen eigentlich ein vertrautes Modell sein (z. B. Gremienarbeit, Forschungsprojekte) - und könnte in seiner Ausweitung durch studentische Partizipation einen Schritt auf die Organisation der Bildungseinrichtung für das 21. Jahrhundert darstellen.

\section{Fazit: Agile Prinzipien in der Hochschule}

Nach einer ersten Erprobungsphase lässt sich festhalten, dass agile Prinzipien in der Hochschule prinzipiell eine Passung haben. Die dargestellten agilen Prinzipien sowie das Rahmenwerk Scrum bringen trotz einer augenscheinlichen Nähe von iterativ angelegten Prozessen zur akademischen Kultur sowohl Herausforderungen wie auch Chancen für die Entwicklung von Lehre und Studium in der Hochschule mit sich. Erste Erfahrungen in beide Richtungen konnten hier aufgezeigt werden, weitere sollen zukünftig systematisch herausgearbeitet und publiziert werden.

Die ersten Monate im Universitätskolleg 2.0 zeigen zudem recht deutlich, inwiefern die Adaption dieses Rahmenwerks und der Methode nicht nur vom Gegenstand der Maßnahme, sondern auch von den beteiligten Akteurinnen und Akteuren und ihrer Haltung abhängig ist. Entsprechend galt und gilt es hier, die vier agilen Prinzipien zwar stetig zu realisieren, doch zugleich auch in ihrer Intensität in Passung mit den jeweiligen Teams und ihrem eigenen Tempo zu bringen, um die jeweiligen Produkte erfolgreich(er) zu entwickeln. Ziel ist es, für das Universitätskolleg 2.0 möglichst alle Teams in ihrer jeweiligen Spezifik im Sinne der agilen Prinzipien zu entwickeln, damit die jeweiligen Maßnahmen entsprechend der Leitidee nachhaltig von den Stakeholdern mitgetragen werden und einen Beitrag zur Verbesserung von Studium und Lehre leisten.
Open Access Dieser Artikel wird unter der Creative Commons Namensnennung 4.0 International Lizenz (http://creativecommons.org/ licenses/by/4.0/deed.de) veröffentlicht, welche die Nutzung, Vervielfältigung, Bearbeitung, Verbreitung und Wiedergabe in jeglichem Medium und Format erlaubt, sofern Sie den/die ursprünglichen Autor(en) und die Quelle ordnungsgemäß nennen, einen Link zur Creative Commons Lizenz beifügen und angeben, ob Änderungen vorgenommen wurden.

\section{Literatur}

Gloger, B. (2016). Scrum. Produkte zuverlässig und schnell entwickeln. München: Hanser.

Hanft, A., Brinkmann, K., Kretschmer, S., Maschwitz, A., \& Stöter, J. (2016). Organisation und Management von Weiterbildung und Lebenslangem Lernen an Hochschulen. Münster: Waxmann.

Hanft, A., Maschwitz, A., \& Stöter, J. (2017). Agiles Projektmanagement an Hochschulen - get the things done. Synergie, 2017/03, 8-15. https://synergie.blogs.uni-hamburg.de/ausgabe-03-beitraghanft-maschwitz-stoeter/. Zugegriffen: 7. Mai 2017.

Mayrberger, K. (2017). Agilität und (Medien-)Didaktik - eine Frage der Haltung? Synergie, 2017/03, 16-19. https://synergie.blogs. uni-hamburg.de/ausgabe-03-beitrag-mayrberger/. Zugegriffen: 7. Mai 2017.

Reddy, A. (2015). The Scrumban [R]Evolution: Getting the Most Out of Agile, Scrum, and Lean Kanban. Reading: Addison Wesley.

Schwaber, K., \& Sutherland, J. (2016). Der Scrum Guide. Der gültige Leitfaden für Scrum: Die Spielregeln. http://www.scrumguides. org/docs/scrumguide/v2016/2016-Scrum-Guide-German.pdf. Zugegriffen: 7. Mai 2017.

Schwaber, K., Sutherland, J., \& Beedle, M. (2001). Agile software development with Scrum. Upper Saddle River: Prentice Hall.

Universitätskolleg (2017). Gestern, heute, morgen. Das Universitätskolleg von 2012 bis 2016. https://www.universitaetskolleg.unihamburg.de/publikationen/schriftenreihe.html. Zugegriffen: 6 . Juli 2017. Universitätskolleg-Schriften Bd. 22.

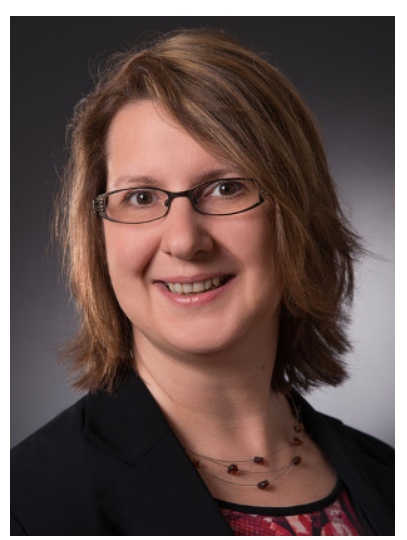

Prof. Dr. Kerstin Mayrberger Beauftragte der Universität Hamburg für Digitalisierung von Lehren und Lernen; Wissenschaftliche Leitung des Universitätskollegs; Professorin mit Schwerpunkt Mediendidaktik am Hamburger Zentrum für Universitäres Lehren und Lernen (HUL). 


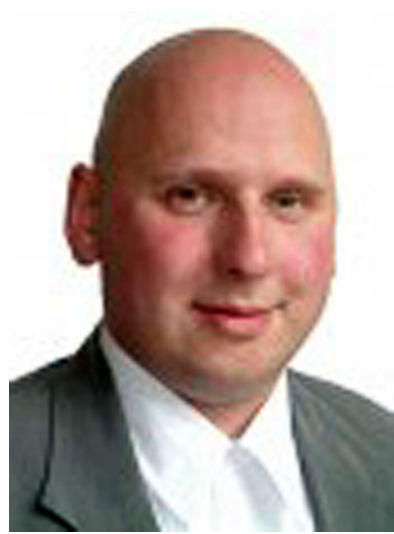

Markus Slobodeaniuk freiberuflicher Unternehmensberater (Organisation-, Prozess-, Kommunikations- und IT-Beratung); Professional Scrum Master/Product Owner/ Developer; seit 2005 an den Hamburger Hochschulen bei Projekten zur Lehrorganisation und Digitalisierung involviert. 